\title{
Detection of very low-frequency oscillations of cerebral haemodynamics is influenced by data detrending
}

\author{
T. Müller ${ }^{1,2}$ \\ J. Timmer ${ }^{1,2}$ \\ M. Reinhard ${ }^{3}$ \\ E. Oehm ${ }^{3}$ \\ A. Hetzel ${ }^{3}$ \\ ${ }^{1}$ Centre for Data Analysis \& Modelling, University of Freiburg, Freiburg, Germany \\ ${ }^{2}$ Department of Physics, University of Freiburg, Freiburg, Germany \\ ${ }^{3}$ Department of Neurology \& Clinical Neurophysiology, University of Freiburg, Freiburg Germany
}

\begin{abstract}
Recent studies were investigated that report spontaneous oscillations of cerebral perfusion in the very low-frequency range $(0.01-0.04 \mathrm{~Hz})$, emphasising details of spectral estimation. The effects of different spectral estimation procedures were compared, using simulated and clinical data. It was shown that data detrending, as used in many studies, can lead to an artifactual peak in the very low-frequency region of estimated power spectra, indicating that the peak cannot be taken as evidence of physiological oscillations. A quantitative, reliable method is described that can be used to assess very low-frequency oscillations. Using the method, very low-frequency oscillations were found in ten out of 17 healthy adults measured with transcranial Doppler laverage frequency, $0.021 \pm 0.007 \mathrm{~Hz}$, mean士 $S D$ ), confirming earlier findings based on visual inspection of data.
\end{abstract}

Keywords-Spectral analysis, Power spectrum, Spectral estimation, Detrending data, Very low-frequency oscillations, $B$ waves

\section{Introduction}

SPECTRAL METHODS based on the mathematical Fourier transformation are a powerful tool for data analysis. Since the advent of the fast Fourier transform algorithm in the 1960s, such methods are computationally affordable and have become increasingly popular in cardio- and cerebrovascular physiology. In this study, we show that spectral methods have intrinsic limitations that need to be taken into account when analysing the spectral properties of cerebral haemodynamics in the very low-frequency range.

The time-course of cerebral perfusion has been studied with a variety of techniques, including magnetic resonance imaging, transcranial Doppler and near infrared spectroscopy. The power spectrum of cerebral perfusion shows spontaneous oscillations in a variety of frequency bands

(a) a pronounced peak at the pulse frequency around $1 \mathrm{~Hz}$ (P-waves)

(b) a broad peak at the respiratorial frequency around $0.3 \mathrm{~Hz}$ (R-waves)

(c) a peak in the so-called 'low-frequency' region around $0.1 \mathrm{~Hz}$ (M-waves).

The link between spectral features $(a)$ and $(b)$ and heart rate and respiration is well established (MALLIANI et al., 1994). The low-frequency $\mathrm{M}$-waves were first observed for arterial blood

Correspondence should be addressed to Dr Jens Timmer; email: jeti@fdm.uni-freiburg.de pressure (MAYER, 1876) and have been linked to sympathetic neural oscillations (PREISS and POLOSA, 1974).

Even slower, very low-frequency oscillations (VLFOs), with frequencies around $0.03 \mathrm{~Hz}$, were observed in cerebral perfusion and thus intracranial pressure. These oscillations were labelled B-waves, as they are assumed to reflect regular changes in the vasomotor tone of cerebral arterioles and thus cerebral blood volume, generated by various brain stem nuclei (LUNDBERG, 1960).

Recent reports of VLFOs have mostly interpreted a peak in the very low-frequency region of estimated power spectra as evidence of corresponding physiological oscillations. In many studies, analysed time series were detrended prior to computation of the power spectrum. However, data detrending, i.e. the removal of the mean or of higher-order polynomials, has been reported to influence estimated power spectra at their lowfrequency end by introducing a spurious peak (HAMMING, 1989).

The purpose of the present study was therefore, first, to analyse the effect of data detrending on the assessment of VLFOs and, secondly, to search for VLFOs of cerebral perfusion in healthy adults, using a robust method.

\section{Methods}

\subsection{Data acquisition}

Seventeen healthy adults were studied $(27 \pm 4$ years $[ \pm S D]$, eight women). Cerebral blood flow velocity (CBFV) in both middle cerebral arteries (MCAs) was measured using $2 \mathrm{MHz}$ transcranial Doppler transducers* attached to a headband. Both 
MCAs were insonated through the temporal bone window after identification according to standard criteria (ARNOLDS and VON REUTERN, 1986). Subjects rested in a supine position in a quiet, dimly lit room. After establishing stable baseline values, a period of $8 \mathrm{~min}$, with subjects awake and breathing spontaneously, was recorded at a sampling rate of $100 \mathrm{~Hz}$.

\subsection{Spectral analysis}

Spectral methods are available in a variety of commercial data analysis software packages, as well as in source code form (PRESSet al., 1992). Mathematical details can be found in BROCKWELL and DAVIS (1991).

The power spectrum $S_{x}(f)$ of a stationary, zero-mean discrete process $x(t), t \in \mathcal{Z}$, is defined as the expected value of the squared modulus of the Fourier transform (FT) $\tilde{x}(f)$ of the process

$$
\begin{aligned}
\tilde{x}(f) & =\lim _{N \rightarrow \infty} \frac{1}{\sqrt{2 N}} \sum_{t=-N}^{N} x(t) \exp (-2 \pi i f t) \\
S_{x}(f) & =\left\langle|\tilde{x}(f)|^{2}\right\rangle
\end{aligned}
$$

Real data $x(t)$ are usually measured at discrete times $t_{i}=i \Delta t$, where $\Delta t$ is the sampling interval; the sampling frequency $f_{S}=1 / \Delta t$. Only a finite number of data sampled at times $t_{i}$, $i=0, \ldots, N-1$, can be made available for analysis. As there is only a finite amount of information in the finite measurement, we cannot expect to infer the power spectrum reliably at all frequencies. Rather, the discrete FT of the measured data

$$
\tilde{x}\left(f_{j}\right)=\frac{1}{\sqrt{N}} \sum_{k=0}^{N} x\left(t_{k}\right) \exp \left(-2 \pi i f_{j} t_{k}\right)
$$

gives independent estimates only at the frequencies

$$
f_{j}=\frac{j}{N \Delta t}=\frac{j f_{S}}{N} \quad j=0, \ldots, N / 2
$$

i.e. from $f_{0}=0$ to the Nyquist frequency $f_{N y q}=f_{N / 2}=f_{S} / 2$, which is the maximum frequency that can be detected using a sampling interval of $\Delta t$. The frequency resolution is

$$
\Delta f=\frac{1}{N \Delta t}=\frac{f_{S}}{N}=\frac{1}{T}
$$

where $T=N \Delta t$ is the measurement interval. Thus, the longer the measurement, the finer the frequency resolution within the Nyquist interval from 0 to $f_{N y q}$.

From the discrete FT $\tilde{x}$, the periodogram $P_{x}$ can be calculated at frequencies $f_{j}$

$$
P_{x}\left(f_{j}\right)=\left|\tilde{x}\left(f_{j}\right)\right|^{2} \quad j=0, \ldots, N / 2
$$

There are two problems that we face if we want to use the periodogram as an estimate of the power spectrum. The first is the problem of periodogram variance. A periodogram (6) has a large variance that does not decrease with increasing numbers of data. As more data are made available, only the frequency resolution of the periodogram increases (5), but the variance stays the same. Thus the periodogram is not a consistent estimator of the power spectrum. To overcome this defect, frequency resolution can be traded for variance in a number of ways.

The second problem of spectral estimation using the periodogram is known as 'leakage'. Briefly, a finite stretch of data is mathematically the product of an underlying infinite time-series and a rectangular window that steps from zero to one and back. Using the convolution theorem (BROCKWELL and DAVIS, 1991), in the FT computed from a finite number of data, power is thus transferred from peaks to adjacent bins. One remedy for this problem is data tapering, i.e. multiplying the given data by a smoothly rising and falling window function.
Both mentioned problems can be overcome by the popular Welch method, which is the spectral estimation method used in most studies reporting VLFOs (GILLER et al., 1999; KUO et al., 1998; ZHANG et al., 1998; OBRIG et al., 2000). It is readily available as function PSD of the MATLAB(c) software package ${ }^{\dagger}$. With this method, $N$ given data points are divided into segments of length $N_{F F T}$, often a power of two. The segments are then individually tapered with a window function, and the periodograms for all segments are computed. Finally, the periodograms of all segments are averaged. Compared with the periodogram, this method reduces both the problem of leakage (by tapering) and the variance of the power spectrum estimate (by averaging). Overlapping segments can further reduce the variance. When $N$ data points are sampled with sampling frequency $f_{S}$, the Welch method gives estimates for the power spectrum at frequencies

$$
f_{j}=\frac{j f_{S}}{N_{F F T}} \quad j=0, \ldots, N_{F F T} / 2
$$

i.e. compared with the original periodogram, the frequency resolution is reduced by a factor of $N / N_{F F T}$. Note that, with the Welch method, the frequency resolution only depends on the sampling frequency $f_{S}$ and on the parameter $N_{F F T}$. With increasing numbers of data, the variance of the power spectrum estimate will be reduced. As an example, with $f_{S}=10 \mathrm{~Hz}$ and $N_{F F T}=1024$, the frequency resolution is approximately $0.01 \mathrm{~Hz}$. The important point is that the frequency (in $\mathrm{Hz}$ ) is the inverse of the length (in seconds) of the segments for which the FT is computed, independent of the total measurement interval.

Periodogram smoothing is a valuable alternative to the Welch method (TIMMER et al., 1996). As the spectrum of almost all real-life processes is smooth, the variance of the spectrum estimate can be reliably reduced by smoothing the periodogram, e.g. with a triangular window. The smoothed periodogram nominally has the same frequency resolution as the initial periodogram, but the effective frequency resolution will be reduced. The width of the smoothing window must be chosen so as to balance the effects of a short window (little reduction in variance, small bias and high effective frequency resolution) and a long window (large reduction in variance, large bias and low effective frequency resolution); see TIMMER et al. (1996).

Estimated power spectra should always be plotted with a logarithmically scaled $y$-axis, as, on a linear scale, the relevant details of smaller peaks will be lost (TIMMER et al., 1996).

\subsection{Data detrending}

The definition of the power spectrum in the preceding Section was given with the proviso that the processes have zero mean. Real data will often not fulfil this condition, but it seems easy to correct for this by subtracting the mean (or 'baseline') of the data. Moreover, there may be a linear trend in the data, and baseline fluctuations can also be more erratic. From the perspective of the ideal measurement that we want to achieve, it is desirable to remove all baseline shifts by detrending. Generalisations of linear detrending to higher-order detrending appear promising.

Such higher-order detrending has been used by many groups that report VLFOs (HosHI et al., 1998; ZHANG et al., 1998; OBRIG et al., 2000). Although the aim of correcting for baseline fluctuations is certainly a reasonable one, the question of the effect of such data preprocessing on the power spectrum estimate appears to have been neglected. HAMMING (1989) warns that a spurious peak at very low frequencies can appear owing to detrending: Detrending reduces the power at the lowest frequencies, 'bending' the estimated spectrum towards zero. The resulting point of inflection is liable to be interpreted as a peak, even if the

\footnotetext{
The MathWorks, Inc., Natick, MA
} 
input was Gaussian white noise. The spurious peak will generally appear at the third frequency bin. The corresponding frequency depends on the resolution of the spectral estimate. In the case of the Welch method, the peak position thus depends on $N_{F F T}(7)$, whereas, in the case of periodogram smoothing, the peak position depends on the length of the time series.

\subsection{Assessment of $V L F O s$}

The power spectrum was computed from 48000 data points $(8 \mathrm{~min}$ at $100 \mathrm{~Hz})$, by the smoothing of the periodogram with a triangular window with a total width of five bins. No detrending was used. The first five frequency bins $(0-0.0083 \mathrm{~Hz})$ were discarded owing to instationarities.
(Note that this mandates the use of periodogram smoothing, as, with the reduced frequency resolution of the Welch method, we would be discarding the frequency region that we are interested in.) In the remaining VLF range from 0.01 to $0.04 \mathrm{~Hz}$, the following operational criterion was used (TIMMER et al., 1996): a local maximum was considered a peak and thus evidence of physiological oscillations if, somewhere to the left (down to frequency 0 ) and somewhere to the right (up to frequency $0.05 \mathrm{~Hz}$ ), the estimated spectrum was significantly smaller, i.e. below the $95 \%$ confidence interval for the peak. For the described smoothing, this means that a peak must be flanked by 'valleys' in which the estimated power is less than $18 \%$ of the peak value. If several peaks were significant, the lowest frequency was chosen.
A
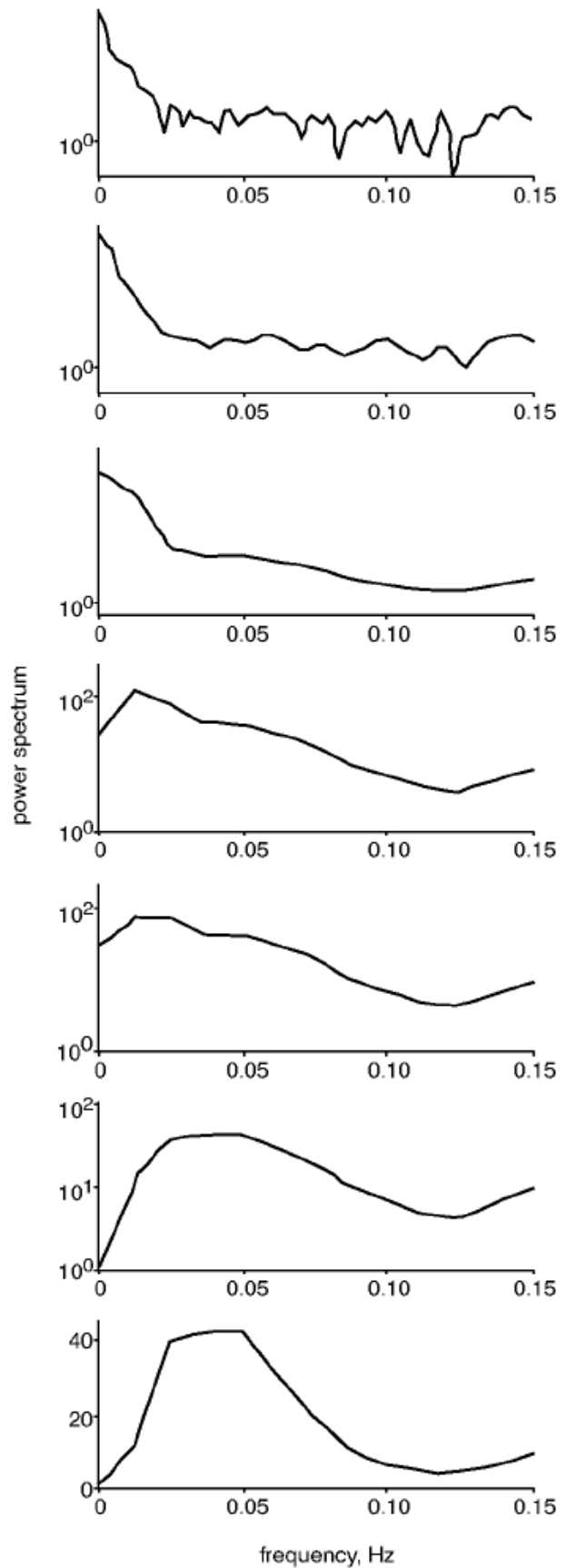
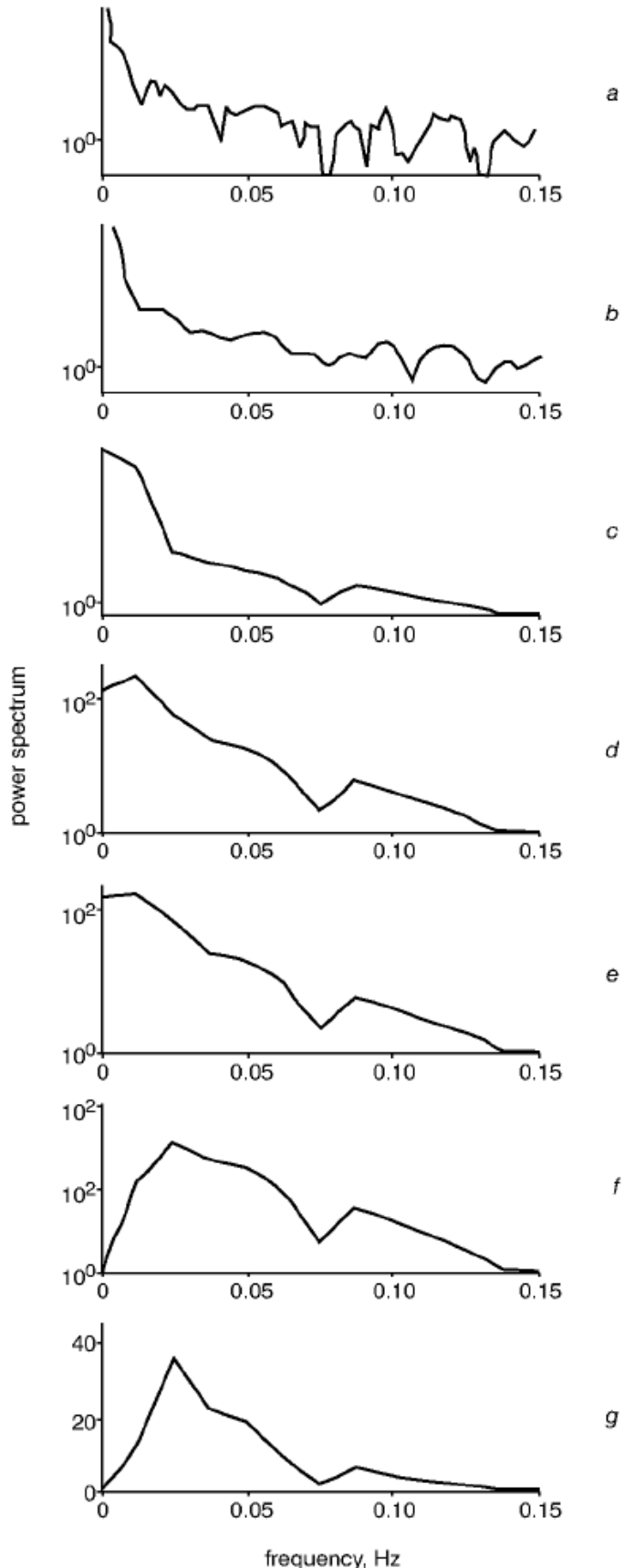

d

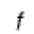

Fig. 1 Estimated spectra for data sets A (measured data) and B (simulated data) in range 0-0.15 Hz. Following spectral estimation methods were used: (a) raw periodogram; (b) smoothed periodogram (triangular window of total width 5 bins; see Section 2.4); (c) Welch method, segment length 8000 bins, no overlapping, no detrending; (d) same as (c), but mean removed from each of six segments; (e) linear trend removed; $(f)$ third-order detrending. All spectra are plotted with logarithmically scaled y-axis. $(g)$ same as (f), but plotted with linearly scaled $y$-axis (shown for ease of comparison with many published spectra) 


\section{Results}

\subsection{How detrending can lead to artifacts}

The effect of detrending on the power spectrum estimate was assessed for two data sets of length $8 \mathrm{~min}$ with a sampling rate of $100 \mathrm{~Hz}$. Set A was the transcranial Doppler recording of a 28-year-old female's left MCA CBFV, arbitrarily selected from the data described in Section 2.1.

Set B was a realisation of a process whose power spectrum mimics two prominent features of the periodograms of the data sets from which recording A was selected

(i) the periodogram rose steeply towards the lower end of the spectrum $(f=0)$, which is the result of instationarities in the data

(ii) there was a broad peak around $1.1 \mathrm{~Hz}$ corresponding to the heart rate.

Thus we chose the sum of a realisation of so-called $1 / f$ noise (TIMMER and KÖNIG, 1995; RAMBALDI and PINAZZA, 1994) and of an $A R[2]$ process (BROCKWELL and DAVIS, 1991). The latter is defined as

$$
x\left(t_{j}\right)=a_{1} x\left(t_{j-1}\right)+a_{2} x\left(t_{j-2}\right)+\varepsilon\left(t_{j}\right)
$$

where $\varepsilon\left(t_{j}\right)$ is Gaussian white noise. The spectrum of the $1 / f$ noise process is proportional to $1 / f^{\beta}$, corresponding to feature (i), and the spectrum of the $A R[2]$ process, which is a damped stochastic oscillator, shows a broad peak at $1.1 \mathrm{~Hz}$ (feature (ii)). Parameters used were $\beta=1.2, a_{1}=1.992, a_{2}=-0.998$, and the simulated sampling frequency was set to $100 \mathrm{~Hz}$, which is the sampling frequency of the measured data.

The rationale for choosing set $\mathrm{B}$ was that with simulated data, we could be absolutely certain that no VLFOs were present, as the spectrum of the simulated process is known (BROCKWELL and DAVIS, 1991). Thus, any peak in the VLF region of the spectrum estimated for set B must stem from the spectral estimation procedure.

Fig. 1 shows the effect of various procedures for spectral estimation on the frequency range from 0 to $0.15 \mathrm{~Hz}$. In line with
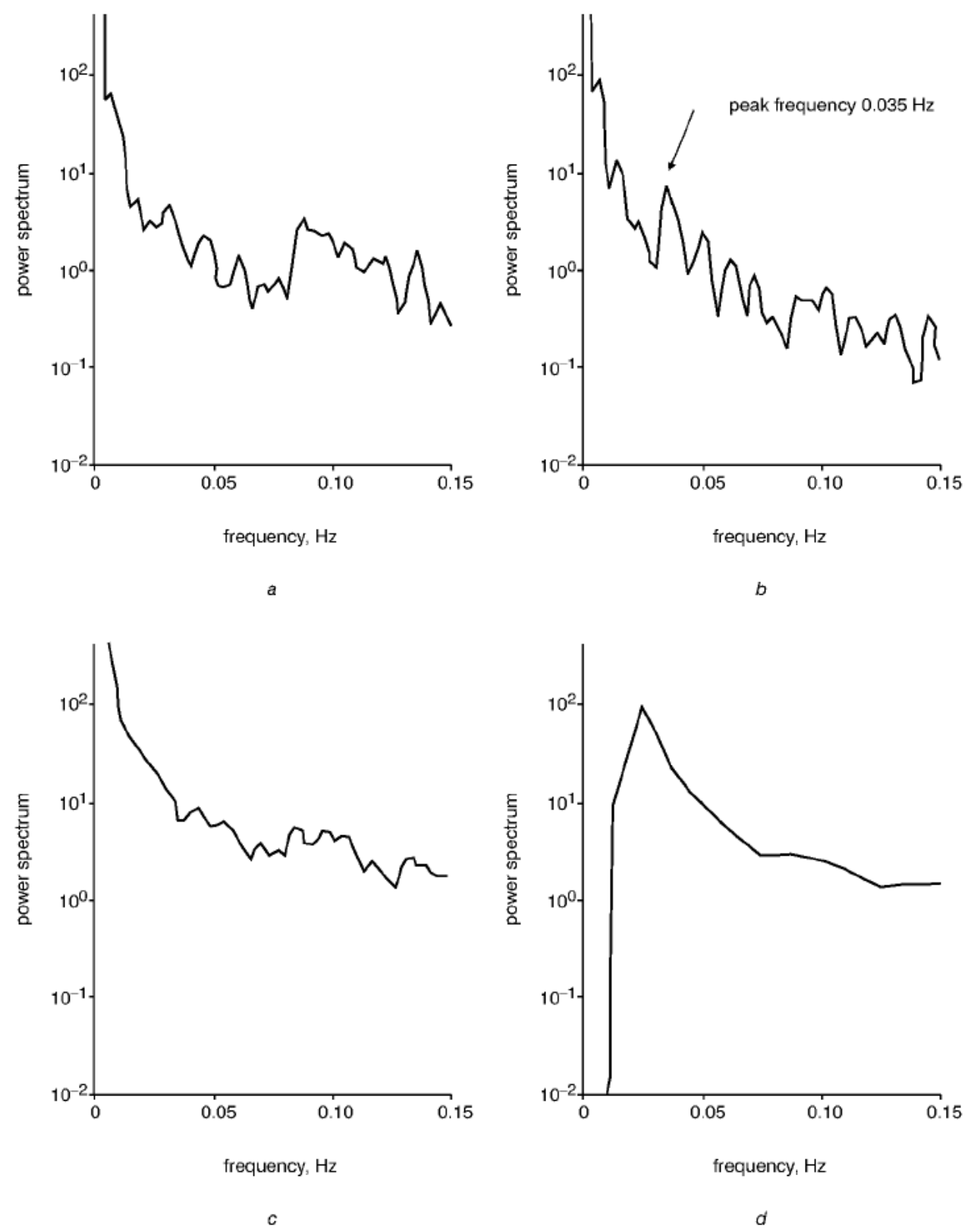

Fig. 2 Estimated spectra plotted in range 0-0.15 Hz. (a) Spectrum showing no VLFOs; (b) spectrum showing VLFOs at 0.035 Hz (assessed using criterion of Section 2.4); (c) average of all 17 power spectra of TCD signals from right MCA CBFV, computed with method of Section 2.4, no detrending (see Fig. Ib); (d) same as (c), but using Welch method with third-order detrending (see Fig. If) 
the mathematical argument mentioned in Section 2.3, a peak in the first few frequency bins appears owing to detrending. The effect is stronger, the higher the order of the polynomial that was subtracted from each data segment in the Welch method: it is already slightly visible with the mean taken out (0th order, Fig. $1 d$ ) and with linear detrending (first order, Fig. 1e), and the effect is quite striking with third-order detrending (Fig. 1f) and even more so on a linear scale (Fig. $1 \mathrm{~g}$ ). In comparison with the Welch method, the smoothed periodogram (Fig. 1b) has a significantly lower variance than the raw periodogram and is not affected by detrending artifacts.

\subsection{Evidence for VLFOs}

All 34 TCD measurements were checked for VLFOs, using the method described in Section 2.4. We found VLFOs in ten out of 17 subjects. Five subjects showed VLFOs in both right and left hemispheres, whereas in five subjects, VLFOs could only be detected unilaterally. The average frequency of the VLFOs was $0.021 \pm 0.007 \mathrm{~Hz}($ mean \pm SD) .

Fig. 2 presents estimated power spectra showing no VLFOs (Fig. 2a) and VLFOs at a frequency of $0.035 \mathrm{~Hz}$ (Fig. 2b). In addition, the average power spectrum of all 17 recordings of right MCA CBFV is shown, estimated without detrending (Fig. 2c) and with third-order detrending (Fig. $2 d$ ). Although averaging smoothes out the VLFOs that are present owing to their different frequencies if no detrending is used, third-order detrending leads to a persistent peak in the VLF region of the estimated spectra.

\section{Discussion}

Our results give reasons for treating some recently reported findings of VLFOs with caution. Linear or third-order detrending prior to spectral analysis has been used by many groups (OBRIG et al., 2000; KUO et al., 1998; YANG et al., 1995; GILLER et al., 1999). Our simulation studies reported here suggest that such spectral estimation procedures do not allow us to separate physiological oscillations in the very lowfrequency range from detrending artifacts. In fact, some Figures in the mentioned studies illustrate the effect described by HAMMING (1989), as a reported VLFO peak appears exactly at the third frequency bin. We believe that our results also shed some light on a question posed by DIEHL and BERLIT (1996), who distinguish two cases of VLFOs or B-waves. We conjecture that true VLFOs should be visible in the time domain, whereas VLFOs detectable solely by frequency analysis may arise from detrending.

Our findings do not, of course, deny the existence of VLFOs in data analysed with detrending. The methodological point is rather that, with detrending, no positive evidence for VLFOs can be obtained from data, even if VLFOs are present.

Some studies put the existence of VLFOs beyond any reasonable doubt, as they are not susceptible to the issues discussed in this paper. Such studies have either argued exclusively in the time domain (DÓRA and KovÁCH, 1981), or they have used spectral estimation procedures carefully (BÄZNER et al., 1995; NEWELL et al., 1992; ELWELL et al., 1999; Li et al., 2000). Our finding of B-waves in ten out of 17 subjects $(59 \%)$ matches well with numbers established by visual inspection of measurements: MAUTNER-HUPPERT et al. (1989) found B-waves in eight out of ten subjects; DROSTE et al. (1994) report B-waves in all subjects measured overnight and estimate that B-waves are present $35-73 \%$ of the time.

We suggest that the method described in Section 2.4 be used to study VLFOs. Data should not be detrended. Rather, the first bins of estimated power spectra should be discarded. In this way, it is possible to assess VLFOs on a methodologically justified, quantitative basis.

\section{Conclusions}

The spectral properties of cerebral perfusion have been studied for many years. Although the interpretation of most parts of the spectrum poses no special problems, the very lowfrequency region, corresponding to frequencies of $0.01-0.04 \mathrm{~Hz}$, is more difficult to interpret, as details of the estimation procedure can have a tremendous effect. Both theoretical considerations and simulation studies support our conclusion that some reports on very low-frequency oscillations in cerebral perfusion in humans are based on insufficient evidence. Data detrending, as performed in many studies, can lead to a peak in the very low-frequency regime that is independent of the existence of any physiological oscillations with corresponding frequencies.

As the popular Welch method is most susceptible to detrending artifacts, we have proposed an alternative procedure of spectral estimation based on periodogram smoothing. Using this method, we formulated a mathematically precise criterion that can be employed to assess the presence or absence of very low-frequency oscillations in a given stretch of data. According to our criterion, we found such oscillations in ten out of 17 healthy young adults whose cerebral blood flow velocity was recorded using transcranial Doppler, confirming earlier findings based on visual inspection of the data. Thus we have shown that a quantitative, methodologically justified procedure based on periodogram smoothing can be used to detect very lowfrequency oscillations of cerebral haemodynamics.

Acknowledgments - T. Müller and M. Reinhard acknowledge the support of the German Ministry of Education and Research $(b m b+f)$.

\section{References}

ARNOLDS, B. J., and von REUTERN, G. M. (1986): 'Transcranial doppler sonography. Examination technique and normal reference values', Ultrasound Med. Biol., 12, pp. 115-123

BÄZNER, H., DAFFERTSHOFER, M., KONIETZKO, M., and HENNERICI, M. G. (1995): 'Modification of low-frequency spontaneous oscillations in blood flow velocity in large- and small-artery disease', $J$. Neuroimag., 5, pp. 212-218

BrockweLL, P. J., and DAVIS, R. A. (1991): 'Time series: theory and methods' (Springer, Berlin, 1991)

DieHL, R. R., and BERLiT, P. (1996): 'Funktionelle Dopplersonographie in der Neurologie' (Springer, Berlin, 1996), p. 27

DÒRA, E., and KovÁCH, A. G. B. (1981): 'Metabolic and vascular volume oscillations in the cat brain cortex', Acta Physiol. Acad. Sci. Hung., 57, pp. 261-275

Droste, D. W., Krauss, J. K., Berger, W., Schuler, E., and BRowN, M. M. (1994): 'Rhythmic oscillations with a wavelength of $0.5-2 \mathrm{~min}$ in transcranial Doppler recordings', Acta Neurol. Scand., 90, pp. 99-104

Elwell, C. E., Springett, R., Hillman, E., and Delpy, D. T. (1999): 'Oscillations in cerebral haemodynamics. Implications for functional activation studies', Adv. Exp. Med. Biol., 471, pp. 57-65

GILLER, C. A., HATAB, M. R., and GILLER, A. M. (1999): 'Oscillations in cerebral blood flow detected with a transcranial doppler index', $J$. Cereb. Blood Flow Metab., 19, pp. 452-459

HAMMING, R. W. (1989): 'Digital filters', 3rd edn (Prentice Hall, Englewood Cliffs, 1989), p. 226

Hoshi, Y., KosaKa, S., XIE, Y., KoHri, S., and TAMURA, M. (1998): 'Relationship between fluctuations in the cerebral hemoglobin oxygenation state and neuronal activity under resting conditions in man', Neurosci. Lett., 245, pp. 147-150 
KuO, T. B., CHERN, C. M., SHENG, W. Y., WONG, W. J., and Hu, H. H. (1998): 'Frequency domain analysis of cerebral blood flow velocity and its correlation with arterial blood pressure', J. Cereb. Blood Flow Metab., 18, pp. 311-318

LI, S.-J., Biswal, B., Li, Z., Risinger, R., Rainey, C., ChO, J.-K., SALMERON, B. J., and StEIN, E. A. (2000): 'Cocaine administration decreases functional connectivity in human primary visual and motor cortex as detected by functional MRI', Magn. Reson. Med., 43 , pp. $45-51$

LUNDBERG, N. (1960): 'Continuous recordings and control of ventricular fluid pressure in neurosurgical practice', Acta Psychiatr. Neurol. Scand., 149, pp. 1-193

Malliani, A., Pagani, M., and Lombardi, F. (1994): 'Physiology and clinical implications of variability of cardiovascular parameters with focus on heart rate and blood pressure', Am. J. Cardiol., 73, pp. 3C-9C

MAUTNER-HuPPERT, D., HABERL, R. L., DiRNAGL, U., VILLRINGER, A., SCHMIEDEK, P., and EINHÄUPL, K. (1989): 'B-waves in healthy persons', Neurol. Res., 11, pp. 194-196

MAYER, S. (1876): 'Studien zur Physiologie des Herzens und der Blutgefäße. V. Über spontane Blutdruckschwankungen', Sächs. Akad. Wiss. Sitz. Math. Natur'w., 74, pp. 281-307

Newell, D. W., Aaslid, R., Stoss, R., and Reulen, H. J. (1992): 'The relationship of blood flow velocity fluctuations to intracranial pressure B waves', J. Neurosurg., 76, pp. 415-421

Obrig, H., Neufang, M., Wenzel, R., Kohl, M., Steinbrink, J., EINHÄUPL, K., and VILLRINGER, A. (2000): 'Spontaneous low frequency oscillations of cerebral hemodynamics and metabolism in human adults', NeuroImage, 12, pp. 623-639

Preiss, G., and PolosA, C. (1974): 'Patterns of sympathetic neuron activity associated with Mayer waves', Am. J. Physiol., 226, pp. $724-730$

Press, W., Flannery, B., Saul, S., and Vetterling, W. (1992): 'Numerical recipes, 2nd edn' (Cambridge University Press, 1992)
RAMBALDI, S., and PINAZZA, O. (1994): 'An accurate fractional Brownian motion generator', Physica A, 208, pp. 21-30

TIMMER, J., and KöNIG, M. (1995): 'On generating power law noise', Astron. Astrophys., 300, pp. 707-710

TIMMER, J., LAUK, M., and DEUSCHL, G. (1996): 'Quantitative analysis of tremor time series', Electroencephalogr. Clin. Neurophysiol., 101, pp. 461-468

YANG, M. W., KuO, T. B., Llin, S. M., CHAN, K. H., and CHAN, S. H. H. (1995): 'Continuous, on-line, real-time spectral analysis of SAP signals during cardiopulmonary bypass', Am. J. Physiol., 268, pp. H2329-H22335

Zhang, R., ZuCkerman, J. H., Giller, C. A., and Levine, B. D. (1998): 'Transfer function analysis of dynamic cerebral autoregulation in humans', Am. J. Physiol., 274, pp. H233-H241

\section{Author's biography}

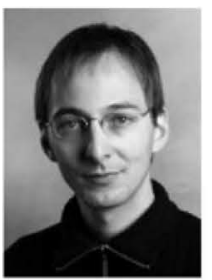

THOMAS MÜLLER studied physics, mathematics, and philosophy in Freiburg, Germany, St. Petersburg, Russia, and at UC Berkeley, CA. He received a diploma in physics, with honours, in 1997 and completed his PhD in philosophy in 2001. He then worked at the Freiburg Center for Data Analysis and Modelling in the group of Jens Timmer, $\mathrm{PhD}$, which specialises in the analysis of time series from biological systems. He is presently an Assistant Professor of Philosophy at the University of Bonn, Germany, specialising in logic, philosophy of science, and theory of action. 